\title{
A Weibull-based damage model for the shear softening behaviours of soil-structure interfaces
}

\author{
Chengchao Guo PhD \\ Associate Professor, School of Civil Engineering, Sun Yat-Sen University, Zhuhai, \\ China; Guangdong Provincial Key Laboratory of Oceanic Civil Engineering, \\ Guangzhou, Guangdong Province, China; Guangdong Provincial Research \\ Center for Underground Space Exploitation Technology, Guangzhou, \\ Guangdong Province, China; Southern Marine Science and Engineering \\ Guangdong Laboratory (Zhuhai), Zhuhai, China; Southern Institute of \\ Infrastructure Testing and Rehabilitation Technology, Huizhou, China \\ Panfeng Guo BEng \\ Graduate student, School of Civil Engineering, Sun Yat-Sen University, \\ Zhuhai, China; Guangdong Provincial Key Laboratory of Oceanic Civil \\ Engineering, Guangzhou, Guangdong Province, China; Guangdong \\ Provincial Research Center for Underground Space Exploitation Technology, \\ Guangzhou, Guangdong Province, China; Southern Marine Science and \\ Engineering Guangdong Laboratory (Zhuhai), Zhuhai, China \\ Liguo Zhao MSC \\ Senior Engineer, CCCC First Highway Consultants Co., Ltd., Xian, China
}

\begin{abstract}
Peiyuan Lin PhD
Associate Professor, School of Civil Engineering, Sun Yat-Sen University, Zhuhai, China; Guangdong Provincial Key Laboratory of Oceanic Civil Engineering, Guangzhou, Guangdong Province, China; Guangdong Provincial Research Center for Underground Space Exploitation Technology, Guangzhou, Guangdong Province, China; Southern Marine Science and Engineering Guangdong Laboratory (Zhuhai), Zhuhai, China (Orcid:0000-0001-7648-6236) (corresponding author: linpy23@mail.sysu.edu.cn)

Fuming Wang PhD

Professor, School of Civil Engineering, Sun Yat-Sen University, Zhuhai, China; Guangdong Provincial Key Laboratory of Oceanic Civil Engineering, Guangzhou, Guangdong Province, China; Guangdong Provincial Research Center for Underground Space Exploitation Technology, Guangzhou, Guangdong Province, China; Southern Marine Science and Engineering Guangdong Laboratory (Zhuhai), Zhuhai, China; Southern Institute of Infrastructure Testing and Rehabilitation Technology, Huizhou, China
\end{abstract}

The shearing mechanical properties of an interface are of great significance to the stability analysis of soil-structure interaction in practice. Based on the theory of damage mechanics, an interface subjected to shearing conditions can be divided into two parts: damaged and undamaged, together bearing the entire shear load. Under the action of shear load, the damaged part gradually increases and the area of the undamaged part simultaneously decreases. Eventually, the interface is completely damaged. The damage variable modelled as a Weibull distribution is introduced to describe the damage evolution of the interface. According to the principle of force equilibrium on the interface, the shear damage softening model is established, which can describe the shear softening behaviours as well as estimate the residual strength of the interface. The physical meaning of each parameter in the model is elaborated and the relationship with normal stress is established. A method to determine each parameter is proposed. Based on the Abaqus software platform, the model is coded into the friction subroutine, and numerical simulation is carried out with reference data. The shear damage model is validated using experimental data and numerical simulation results.

\section{Notation}

A total area of shear interface

$A_{1} \quad$ area of undamaged part of shear interface

$A_{2} \quad$ area of damaged part of shear interface

$a_{p}, b_{p}$ fitting coefficient of peak shear displacement curve

$C_{p} \quad$ cohesion when the interface reaches the peak strength

$C_{r} \quad$ cohesion when the interface reaches the residual strength

$C_{s} \quad$ cohesion at interface yield

$D \quad$ damage variable

G nominal interfacial shear stiffness

F $\quad$ shear cell strength

$F_{0}, m$ Weibull distribution parameters

$k \quad$ interface stiffness coefficient

$R^{2} \quad$ linear goodness of fit value

$s$

$S_{p}$

$s^{*}$

$\sigma_{n}$

$\tau$

$\tau_{1}$

$\tau_{2}$ $\tau_{p} \quad$ peak shear strength

$\tau_{r} \quad$ residual strength

$\tau_{s} \quad$ yield strength

$\phi_{p} \quad$ internal friction angle when the interface reaches the peak strength

$\phi_{r} \quad$ internal friction angle when the interface reaches the residual strength

$\phi_{s} \quad$ internal friction angle at interface yield

\section{Introduction}

The interface between soil and structure widely exists in infrastructure construction engineering; it is an important research subject for geotechnical engineering, and is also the key to solving the interaction between soil and structure in practical engineering. The interface is formed by the contact of two materials with different properties. To study the interaction between soil and structure, besides correctly understanding the mechanical properties of the two materials themselves, it is also necessary to study the mechanical properties of the interface that are different from the two materials. Since the middle of the last century, many scholars have begun to study the mechanical 
properties of the interface between soil and structure. At present, the research content mainly focuses on the interface deformation law of concrete, steel, wood and soil with different surface roughness and different soil moisture content. The research methods are mainly divided into experimental research, theoretical analysis and numerical simulation (Cheng et al., 2018b; Clough and Duncan, 1971; Li et al., 2015; Potyondy and Eng, 1961; Uesugi and Kishida, 1986; Xiong et al., 2018). The general idea of the study is to obtain the stress-strain curves of different soil and structure interfaces through relevant laboratory tests. According to the curves and using the theory of mechanics and mathematics, the mathematical models describing the constitutive relations of different interfaces are established, and verified by numerical simulation. Then, the mathematical model is applied to practical projects, so as to guide the engineering practice.

The shear test is an effective method to obtain the interfacial shear deformation characteristics. However, the mechanical properties of materials are complex and the number of tests is limited, and the test results have a narrow scope of application. Therefore, it is necessary to establish an interface shear model based on the limited test results to predict the interface shear mechanical properties. Existing research results indicate that there are two types of interface surface shear, namely strain hardening and strain softening (Desai and Ma, 1992; Long et al., 2017; Yin et al., 1994). Clough and Duncan (1971) established an interface hyperbolic constitutive model based on the test results, which is suitable for strain hardening problems. Because the model parameters are few and easy to determine, it is widely used. Some scholars have also modified the Clough model to try to solve the strain softening problem and make up for some of the shortcomings of the model (Hu et al., 2017). Based on the results of shear tests on the interface between soil and concrete, Yin et al. believed that the shear deformation at any point of the interface is rigid and plastic, and established an interface rigid plastic model, which is suitable for strain hardening. The thickness element is used for simulation, but the thickness of the contact surface is complicated and subjective, which affects further promotion (Yin et al., 1994). At the same time, some experimental results show that the interface is of the strain softening type (Hu et al., 2017; Long et al., 2017). By introducing the damage mechanics theory, the interface damage softening model is established. The model not only has a clear physical meaning, but can also reflect the shear softening characteristics of the interface, which has good applicability. However, it is difficult to determine the values of strain and shear band thickness when using the relationship between stress and strain. Based on Weibull distribution, Cao et al. (2012) established the shear softening model with shear stress and shear displacement as parameters, which made up for the deficiency of establishing a damage model with stress and strain as parameters, but did not study the method of determining parameters in the model in detail and did not propose specific values. The realisation method limits its further promotion. Long et al. (2017) established a damage strain softening model, which can reveal the shear softening deformation law of the interface. But because of the concept of shear thickness ratio, it is difficult to determine the value. Cheng et al. (2018a) established the shear stress-strain displacement relationship of the reinforced-soil interface. However, the ratio of shear displacement to interface length was used for the shear strain, which was different from using the shear band to calculate the strain. Further demonstration is needed. At present, most elastic-plastic models only predict the strain-hardening relationship and cannot describe the strain-softening behaviour of the interface (Long et al., 2017). The key to establish a damage model is to determine the law of damage evolution. Existing studies have shown that the probability of shear failure mainly obeys the normal distribution, log-normal distribution and Weibull distribution (Cao et al., 2012; Desai and Ma, 1992). The Weibull distribution is more adaptable in describing the internal damage of rock and soil materials, so it is the most widely used.

In conclusion, the interface shear damage model based on Weibull distribution has good applicability in describing the strain softening characteristics of the interface. The parameters have clear physical meaning, theoretical basis and practical value. However, there is less current research on the parameter determination method and numerical realisation of the model, which is the focus of this paper. To study the mechanism of interfacial shear, based on the damage theory and previous research results, this paper derives the interface shear damage softening model, analyses the parameter determination method in detail and puts forward the numerical implementation method of the model, which provides reference for the further promotion of the theory.

\section{Shear damage softening model of the interface based on Weibull distribution}

Basic assumptions of the interface shear damage model For the strain-softening interface, the shear deformation process of the interface between soil and structure has obvious stages under the action of load. After the interfacial shear stress reaches the peak value, the strain softening will occur and finally enter the residual strength stage. In the process of shear deformation, the soil is the main carrier of deformation, and the soil properties affect the strength of the interface. Since the general shear test obtains the shear stress-shear displacement relationship curve and the complexity of the shear strain value, this paper establishes the interface shear stress-shear displacement constitutive model according to the method proposed by Cao et al. (2012). To introduce the theory of damage mechanics and establish the interface shear damage softening model, the following basic assumptions must be made first.

- The soil is an isotropic material, which satisfies the continuum theory.

- Under the action of shear stress, the interface is composed of the damaged part and the undamaged part. The damaged part still has a certain strength, and both bear the shear stress.

- There is a critical damage value on the interface. When the shear stress reaches the critical damage value, the interface 
begins to be damaged. When undamaged, the shear stress and shear displacement obey the linear elastic relationship.

- In the process of shear deformation, the normal stress of the damaged and undamaged parts of the interface is equal to the nominal normal stress on the interface.

\section{Establishment of the damage variable and damage softening model}

Assuming that the total area of the shear surface is $A$, the area of the undamaged part is $A_{1}$, and the area of the damaged part is $A_{2}$. The damage variable $D$ is defined as the ratio of the damaged area to the total area of the shear surface, which represents the deterioration of the bonding element of the interface during the shearing process.

1. $A=A_{1}+A_{2}$
6. $s=s^{*}$

Because the shear strength is completely borne by the damaged part when the interface is completely damaged $D=1$, so $\tau_{2}$ is equal to the residual strength.

7. $\tau_{2}=\tau_{r}$

where $\tau_{r}$ is the residual strength. Substituting Equation 5, Equation 6 and Equation 7 into Equation 4, one can get:

8. $\tau=G s(1-D)+\tau_{r} D$

\section{Establishment of the damage softening model based on Weibull distribution}

To determine the interface damage softening model, according to the method proposed by Cao et al. (2012), it is assumed that the interface is composed of numerous shear cells. The damage variable $D$ is a function of the shear cell strength, and the shear cell strength obeys the Weibull distribution of two parameters. Therefore, the damage variable $D$ can be expressed as:

while $D=1$ indicates complete interface damage. Regardless of the influence of the shear rate on the interface, the shear strength on the interface is borne by the undamaged part and the damaged part. From the balance of forces.

3. $\tau A=\tau_{1} A_{1}+\tau_{2} A_{2}$

where $\tau$ is the shear stress on the interface; $\tau_{1}$ is the shear stress on the undamaged part of the interface; and $\tau_{2}$ is the shear stress on the damaged part of the interface. Substituting Equation 1 and Equation 2 into Equation 3, one can get

4. $\tau=\tau_{1}(1-D)+\tau_{2} D$

For the undamaged part of the interface, the shear stress and relative shear displacement obey the linear elastic relationship.

5. $\tau_{1}=G s^{*}$

where $G$ is the nominal shear stiffness of the interface, and $s^{*}$ is the relative shear displacement of the undamaged part. According to the strain equivalence principle proposed by Lemaitre (1984), in establishing the damage constitutive equation, the relative displacement after a damage can be used to replace the nominal displacement in the constitutive relationship of the undamaged part - that is, the relative shear displacement of the undamaged part is equal to the relative displacement $s$ on the interface.
9. $D\left\{\begin{array}{cc}0 & F \leq 0 \\ \int_{0}^{F} P(x) d x=1-\exp \left[-\left(\frac{F}{F_{0}}\right)^{m}\right] & F>0\end{array}\right.$

where $m$ and $F_{0}$ represent Weibull distribution parameters, and $F$ represents the strength of shear cells, which is determined by Equation 10:

10. $F=\tau_{1}-\tau_{s}=G s-\tau_{s}$

where $\tau_{s}$ represents the shear stress when the interface yields. Substituting Equation 9 and Equation 10 into Equation 8, the interface shear damage softening model based on Weibull distribution can get (Cao et al., 2012):

$\tau=\left\{\begin{array}{cr}G s & G s \leq \tau_{s} \\ \left(G s-\tau_{r}\right) \exp \left[-\left(\frac{G s-\tau_{s}}{F_{0}}\right)^{m}\right]+\tau_{r} & G s>\tau_{s}\end{array}\right.$

\section{Determination of the model parameters}

It can be seen from Equation 11 that there are five parameters in the interface shear softening model: the shear stiffness $G$, yield strength $\tau_{s}$, residual strength $\tau_{r}$, and Weibull distribution parameters $m$ and $F_{0}$, and each parameter has a clear physical 
meaning. The key to the interface constitutive model is to determine the values of the five parameters.

\section{The determination method of each parameter and the} influence of Weibull distribution parameters $m$ and $F_{0}$ on the model

The five parameters in the model can be obtained from the shear stress-shear relative displacement curve, where the shear stiffness $G$ is the slope of the initial stage of the curve, and the yield strength $\tau_{s}$ and residual strength $\tau_{r}$ can be directly obtained from the curve. The shear stress-shear displacement curve has the characteristics of strain softening, and there is a peak point. When the shear stress reaches the peak point, the stress increment is zero - that is:

12. $\left.\frac{\partial \tau}{\partial s}\right|_{s=s_{p}, \tau=\tau_{p}}=0$

13. $\tau_{p}=\left(G s_{p}-\tau_{r}\right) \exp \left[-\left(\frac{G s_{p}-\tau_{s}}{F_{0}}\right)^{m}\right]+\tau_{r}$

Both $m$ and $F_{0}$ are solved by Equation 11, Equation 12 and Equation 13 simultaneously. The differential of the compound function is involved in the solution, and the computation process is long. The Mathematica software is used to solve the problem. Therefore, $m$ and $F_{0}$ can be expressed as:

14.

$$
m=\frac{G s_{p}-\tau_{s}}{\left(G s_{p}-\tau_{r}\right) \ln \frac{G s_{p}-\tau_{r}}{\tau_{p}-\tau_{r}}}
$$

15

$$
F_{0}=\left(G s_{p}-\tau_{s}\right)\left(\ln \frac{G s_{p}-\tau_{r}}{\tau_{p}-\tau_{r}}\right)^{-1 / m}
$$

It can be seen from Equation 14 and Equation 15 that the Weibull distribution parameters $m$ and $F_{0}$ are not only functions of the shear stiffness $G$, yield strength $\tau_{s}$, and residual strength $\tau_{r}$, but also related to the peak shear strength $\tau_{p}$ and peak shear displacement $S_{p}$. It also has a certain influence on the interface shear characteristics. To study the influence of the Weibull distribution parameters $m$ and $F_{0}$ on the interface shear softening model, the experimental data of Cheng et al. (2017) were used to calculate the Weibull distribution parameters when the normal stress was $200 \mathrm{kPa}$. The result is $m=0.72, F_{0}=53$. By changing the value of a single variable $m$ and $F_{0}$, the interface shear stress-shear displacement curve is obtained, as shown in Figure 1 and Figure 2. It can be seen from the Figure 1 and Figure 2 that the change of Weibull distribution parameters $m$ and $F_{0}$ does not affect the linear elastic part of the interface shear stress-shear displacement curve, but has an obvious influence on the nonlinear curve part, which changes the shape of the curve. As the values of $m$

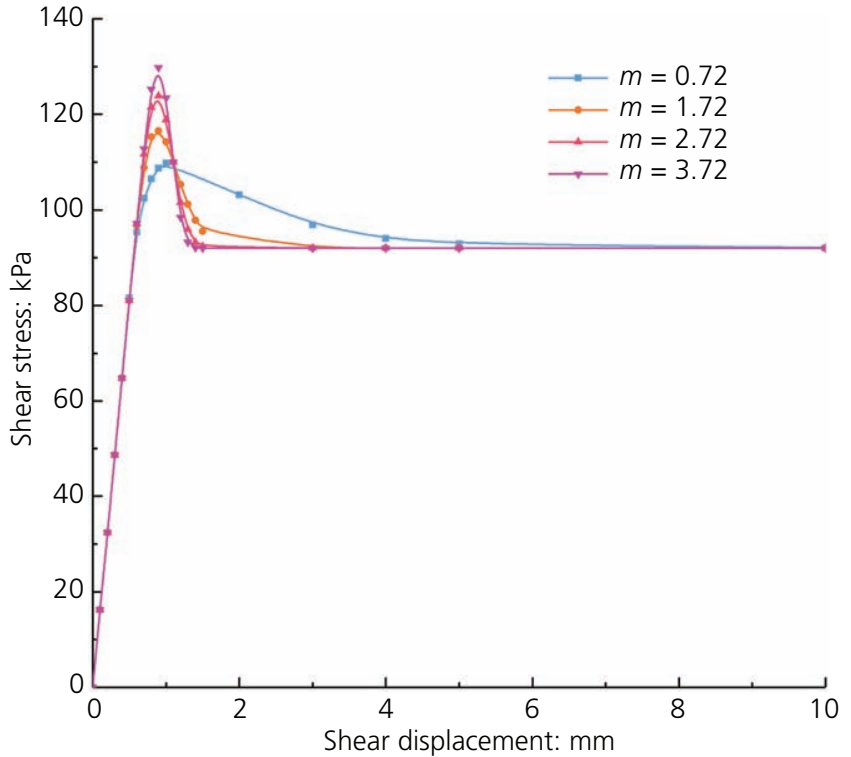

Figure 1. Influence of parameter $m$ on shear damage softening model when $F_{0}=53$

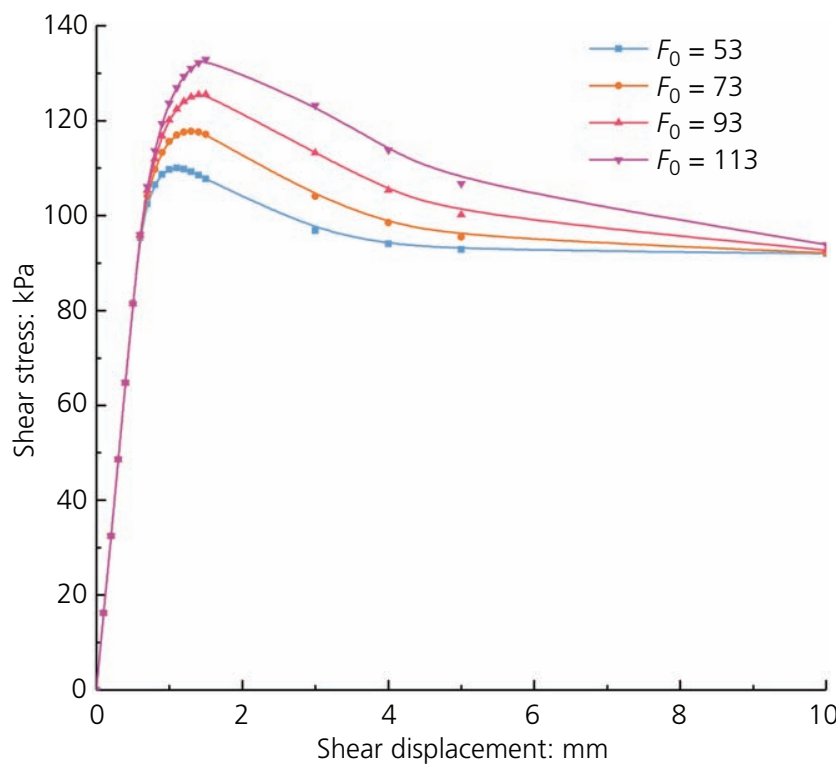

Figure 2. Influence of parameter $F_{0}$ on shear damage softening model when $m=0.72$

and $F_{0}$ increase, the peak shear stress also increases gradually. Therefore, the accuracy of the Weibull distribution parameters $m$ and $F_{0}$ has an important influence on the application of the interface shear damage softening model.

\section{Determination of parameters under different normal} stresses

The interface shear softening model established in this paper has five parameters. Among them, the Weibull distribution parameters 
$m$ and $F_{0}$ are functions of the shear stiffness $G$, yield strength $\tau_{s}$, residual strength $\tau_{r}$, peak shear strength $\tau_{p}$ and the corresponding peak shear displacement $s_{p}$. Therefore, the key is to determine the shear stiffness $G$, yield strength $\tau_{s}$, residual strength $\tau_{r}$, peak shear strength $\tau_{p}$ and the corresponding peak shear displacement $s_{p}$.

A large number of experimental results have shown that the shear stiffness at the initial linear stage of the shear stress-shear displacement curve is less affected by the normal stress, while the yield strength $\tau_{s}$, residual strength $\tau_{r}$, peak shear strength $\tau_{p}$ and the corresponding peak shear displacement $s_{p}$ change with the change of the normal stress (Chen et al., 2018; Hu et al., 2017; Kong et al., 2017; Wang et al., 2019a; Xiong et al., 2018). Under different normal stresses, the values are different. Since the normal stress value taken in the test is limited, and the normal stress value of the interface gradually changes with the buried depth of the structure in actual engineering, it is necessary to use limited test data to establish a universally applicable interface constitutive model. The goal is to establish the relationship between the four parameters of interface yield strength $\tau_{s}$, residual strength $\tau_{r}$, peak shear strength $\tau_{p}$ and corresponding peak shear displacement $s_{p}$ and normal stress. This paper collected and counted a total of 89 shear stress-shear displacement curves of 23 different interfaces in the literature, involving clay, sand, coarse-grained soil, breccia, frozen soil and concrete, steel plate and geogrid interface, and obtained the yield strength $\tau_{s}$, residual strength $\tau_{r}$, peak shear strength $\tau_{p}$ and corresponding peak shear displacement $s_{p}$ of each curve (Chen et al., 2016, 2018; Cheng et al., 2017, 2018b; Hu et al. 2017, 2018; Kong et al., 2017; Li et al., 2015; Wang et al., 2019a, 2019b; Xiong et al., 2017, 2018). A linear fit between each parameter and the normal stress is performed to obtain a linear goodness of fit value $R^{2}$, a histogram of the cumulative frequency distribution of $R^{2}$ is drawn, and the fitting results are shown in Figure 3. It can be seen

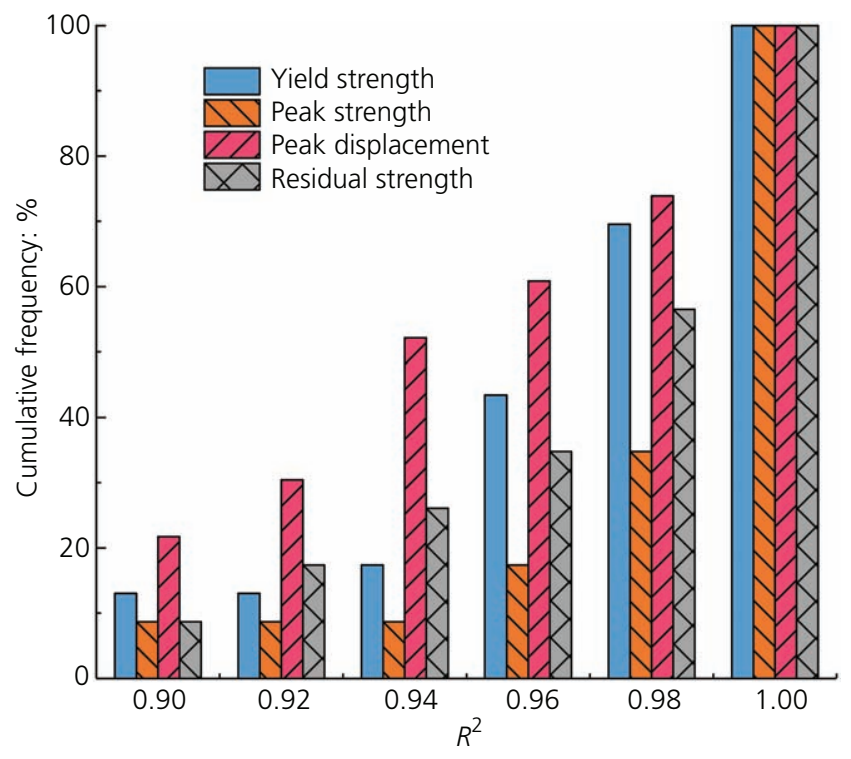

Figure 3. Cumulative frequency distribution of linear goodness of fit from Figure 3 that the average goodness of fit of the yield strength $\tau_{s}$, peak shear strength $\tau_{p}$, peak shear displacement $s_{p}$ and residual strength $\tau_{r}$ are $0.93,0.98,0.90$, and 0.96 , and the proportions greater than 0.9 are $87 \%, 91 \%, 79 \%$ and $91 \%$, respectively. Each parameter has a linear relationship with the normal stress of the interface, and the fitting effect is good. Combined with the Mohr-Coulomb failure criterion, the corresponding empirical formula for each parameter can be written as:

16. $\tau_{s}=\sigma_{n} \tan \phi_{s}+C_{s}$

17. $\tau_{r}=\sigma_{n} \tan \phi_{r}+C_{r}$

18. $\tau_{p}=\sigma_{n} \tan \phi_{p}+C_{p}$

19. $s_{p}=a_{p} \sigma_{n}+b_{p}$

where $\sigma_{n}$ represents the normal stress applied to the interface; $C_{s}$ and $\phi_{s}$ represent the cohesive force and internal friction angle when the interface reaches the interface yields; $C_{r}$ and $\phi_{r}$ represent the cohesive force and internal friction angle when the interface reaches the residual strength; and $C_{p}$ and $\phi_{p}$ represent the cohesion and internal friction angle when the interface reaches the peak strength. $a_{p}$ and $b_{p}$ are the curve fitting coefficients of the peak shear displacement.

The relationship between the five parameters and the normal stress is established above, which makes the model more universal. In practical application, the finite test results can be fitted and the yield strength $\tau_{s}$, residual strength $\tau_{r}$, peak shear strength $\tau_{p}$ and the corresponding peak shear displacement $s_{p}$ under arbitrary normal stress can be calculated according to Equations 16, 17, 18 and 19. According to Equations 14 and 15, the Weibull distribution parameters $m$ and $F_{0}$ are obtained. Finally, the calculated value is substituted into Equation 11 to obtain the interface shear stress-shear displacement curve under any normal stress.

\section{Model validation}

To verify the accuracy of the derived interface shear softening model, two common contact interfaces are selected in this paper, which are clay-concrete interface and gravelly sand-steel interface. The shear stiffness, yield strength, residual strength, peak shear strength and corresponding peak shear displacement under different normal pressures are extracted from the test curves of Cheng et al. (2017) and $\mathrm{Hu}$ et al. (2018b). The shear stress-shear displacement curves are calculated and compared with the test results. The results are shown in Figure 4. It can be seen that the interface shear softening model established in this paper has a good fitting effect with the experimental data. It not only reflects the change of residual strength, but also reflects the influence of normal pressure, which proves the 


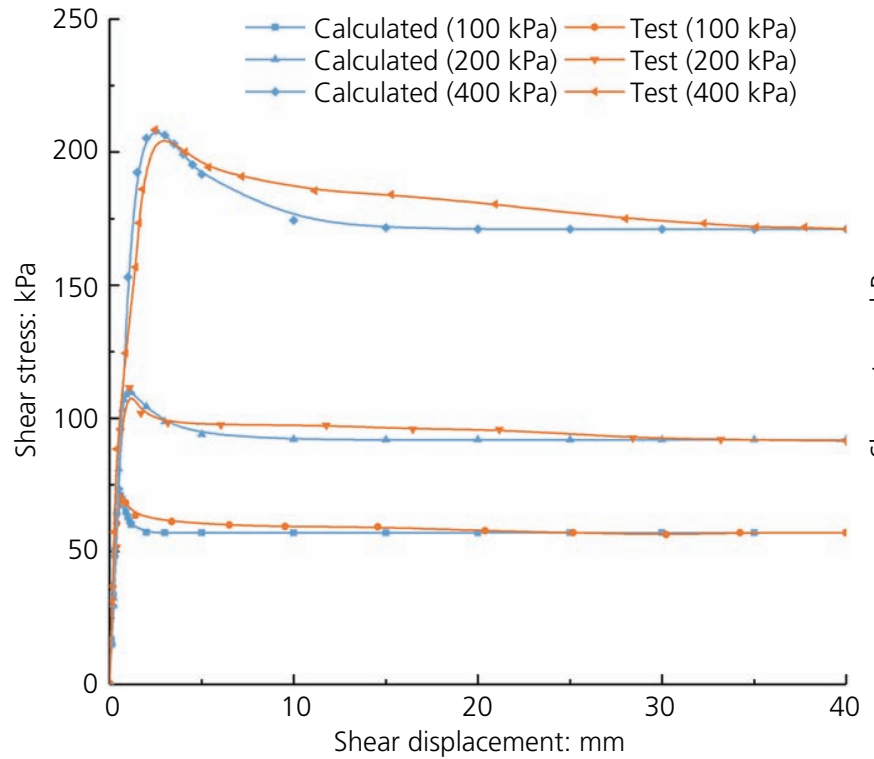

(a)

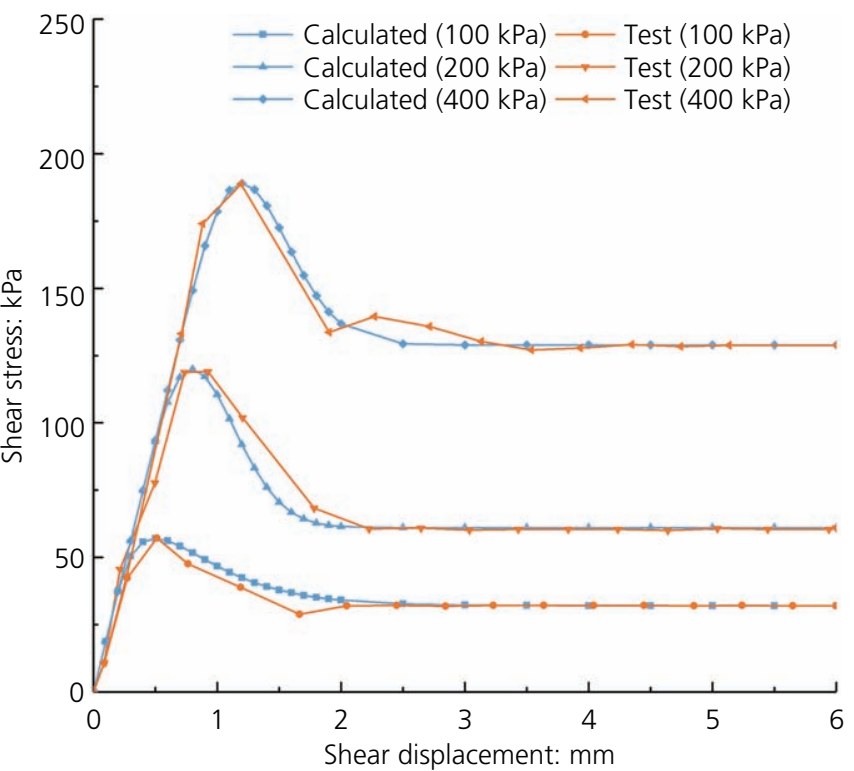

(b)

Figure 4. Comparison of model calculation results and test results: (a) clay-concrete interface; (b) gravelly sand-steel interface

correctness of the interface shear softening model. Figure 5 shows the curves of the damage variable $D$ with shear displacement under different normal stresses at the clay-concrete interface. It can be seen

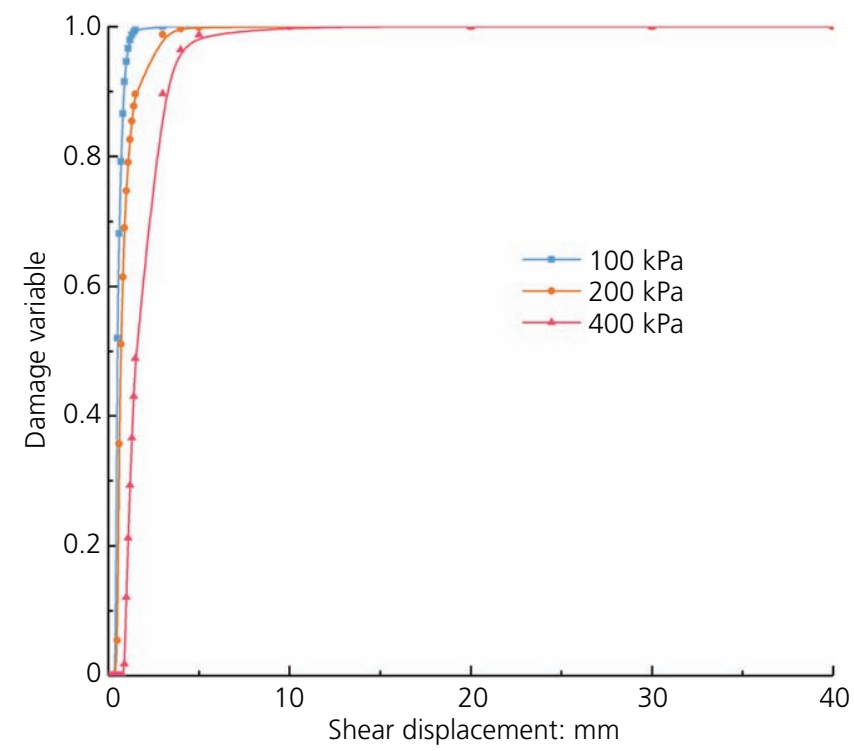

Figure 5. Variation curve of damage variable $D$ with shear displacement under different normal stresses from the curve that with the increase of shear displacement, the damage variable $D$ gradually increases from zero, and finally approaches 1 . At this time, the interface shear element is completely damaged and the interface reaches the residual shear strength. By processing the test data and performing linear fitting, the degree of fit is all greater than 0.98 , and each parameter has a linear relationship with the interface normal stress, which proves the accuracy of Equations 16, 17, 18 and 19. The basic parameter results obtained after processing the data are shown in Table 1 .

\section{Model implementation method and verification based on Abaqus software}

Model implementation method based on Abaqus software

The ideal elastic-plastic Coulomb model is adopted by default in Abaqus software for interface analysis, which cannot simulate the shear softening characteristics of the interface. However, the friction subroutine interface is provided to customise the friction model. In this paper, the FRIC subroutine is used for secondary development, and the established interface shear softening model is written into a subroutine to realise the numerical simulation of the interface. In the FRIC subroutine, only the friction characteristics of the interface need to be considered, the interface stiffness coefficient is given, and the shear stress on the interface is updated according to the slip deformation increment. Abaqus

Table 1. Basic parameters of the test: (1) clay-concrete interface; (2) gravelly sand-steel

\begin{tabular}{|c|c|c|c|c|c|c|c|c|c|}
\hline & G: $\mathrm{kN} \mathrm{m}^{-3}$ & $\mathrm{C}_{s}: \mathrm{kPa}$ & $\varphi_{s}:{ }^{\circ}$ & $C_{r}: \mathrm{kPa}$ & $\varphi_{r}: \circ$ & $C_{p}: \mathrm{kPa}$ & $\varphi_{p}:^{\circ}$ & $a_{p}: \mathrm{mm} \mathrm{kPa}^{-1}$ & $b_{p}: \mathrm{mm}$ \\
\hline (1) & 162000 & 24 & 15 & 17.5 & 21 & 22 & 25 & 0.005 & 0.08 \\
\hline (2) & 187000 & 15.5 & 16.6 & 0 & 17.7 & 22.5 & 23.1 & 0.0023 & 0.3 \\
\hline
\end{tabular}


A Weibull-based damage model for the

shear softening behaviours of

soil-structure interfaces

Guo, Guo, Zhao, Lin and Wang

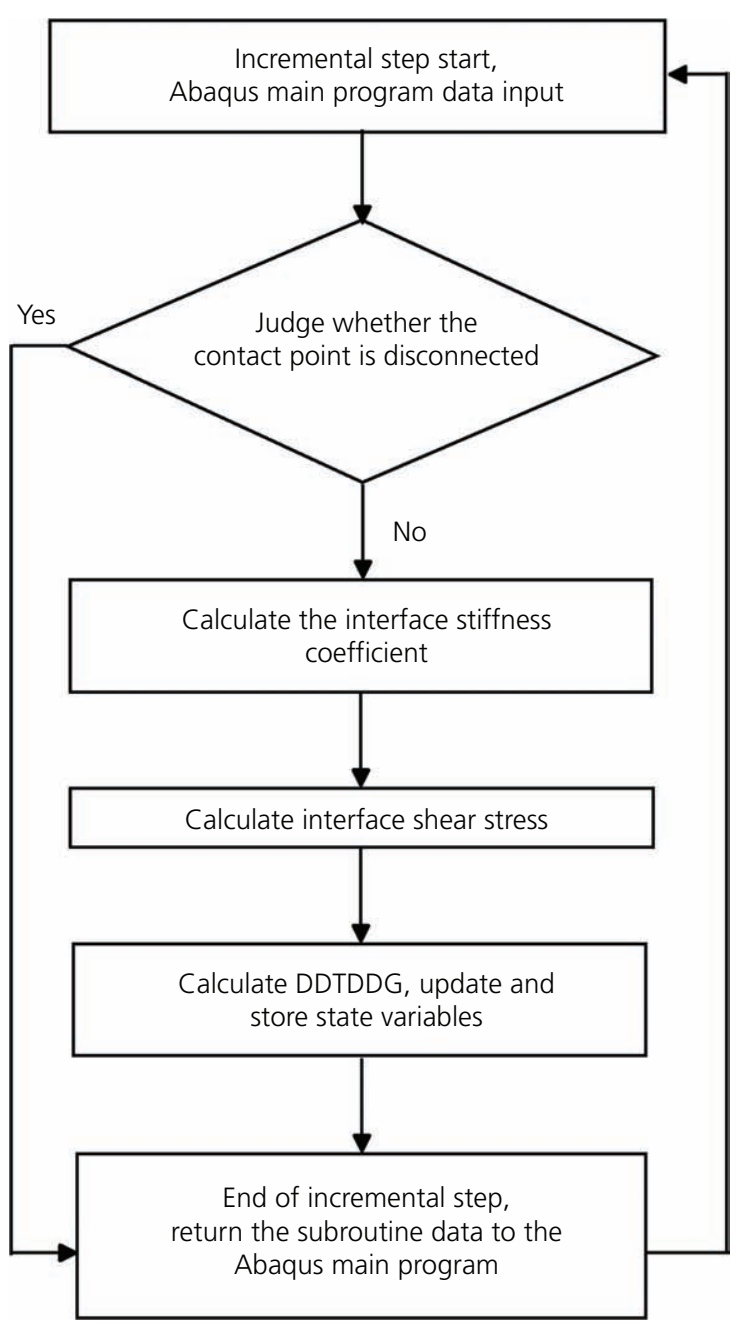

Figure 6. Flow chart of the FRIC subroutine implementation of the interface shear softening model

can judge whether the interface is disconnected. The implementation flow of the FRIC subroutine is shown in Figure 6. In each small increment step, the default shear stress is linearly related to the shear displacement. The partial derivative of shear stress to shear displacement in Equation 11 is calculated to obtain the interface stiffness coefficient at the beginning of the incremental step. The result is shown in Equation 20.

$$
k=\frac{\partial \tau}{\partial s}=G\left\{\exp \left[-\left(\frac{G s-\tau_{s}}{F_{0}}\right)^{m}\right]\right\}
$$

20

$$
\left[\frac{m\left(\tau_{\mathrm{r}}-G s\right)\left(\frac{G s-\tau_{\mathrm{s}}}{F_{0}}\right)^{m-1}}{F_{0}}+1\right]
$$

where $k$ is the interface stiffness coefficient.
Numerical verification of direct shear test based on the FRIC subroutine

To verify the validity of the FRIC subroutine, a two-dimensional finite-element contact model is established according to the test results of Wang et al. (2020). The model is composed of two parts: the upper part is a sand body with a size of $300 \times 150 \mathrm{~mm}$, and the lower part is a polymer with a size of $400 \times 150 \mathrm{~mm}$. The vertical displacement of the bottom of the model and the horizontal displacement of both sides of the sand body are constrained. The $100 \mathrm{kPa}$ load is applied to the top of the sand body, and the horizontal leftward shear displacement is applied on the right side of the polymer. The displacement value is $30 \mathrm{~mm}$. The displacement is applied to simulate the interfacial shearing process. The elastic-plastic model is adopted for the upper sand body. The elastic modulus is $20 \mathrm{MPa}$, Poisson's ratio is 0.2 , and the internal friction angle is $32^{\circ}$. The linear elastic model is used for the lower polymer, with an elastic modulus of $10 \mathrm{MPa}$ and a Poisson's ratio of 0.2. Hard contact is adopted in the normal direction and the FRIC subroutine is used for the tangential direction. The values of input parameters of the numerical model are shown in Table 2, and the schematic diagram of the model is shown in Figure 7.

\section{Analysis of numerical simulation results \\ Shear stress-shear displacement curve}

The shear stress-shear displacement curve on the interface is extracted and compared with the experimental data in the literature and the calculation result of the constitutive model, as shown in Figure 8. The calculation results of the FRIC subroutine are consistent with the results obtained from the experimental data and the constitutive model, and the shear softening characteristics

Table 2. Parameter value of the finite element calculation example

\begin{tabular}{lcccc}
$\begin{array}{l}\text { Shear } \\
\text { stiffness: } \\
\text { kN m }^{-3}\end{array}$ & $\begin{array}{c}\text { Yield } \\
\text { strength: } \\
\mathbf{k P a}\end{array}$ & $\begin{array}{c}\text { Peak } \\
\text { strength: } \\
\mathbf{k P a}\end{array}$ & $\begin{array}{c}\text { Peak } \\
\text { displacement: } \\
\mathbf{m m}\end{array}$ & $\begin{array}{c}\text { Residual } \\
\text { strength: } \\
\mathbf{k P a}\end{array}$ \\
\hline 6100 & 28 & 35 & 8 & 32
\end{tabular}

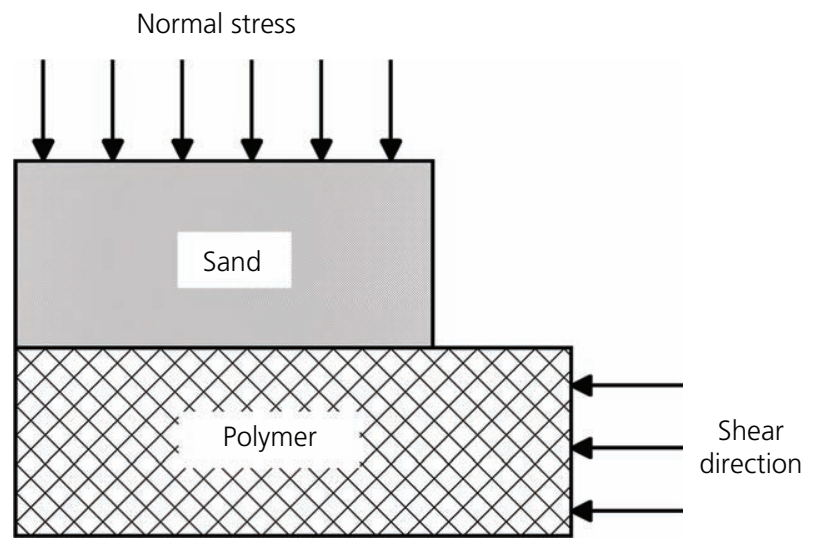

Figure 7. Schematic diagram of the numerical model of the direct shear test 


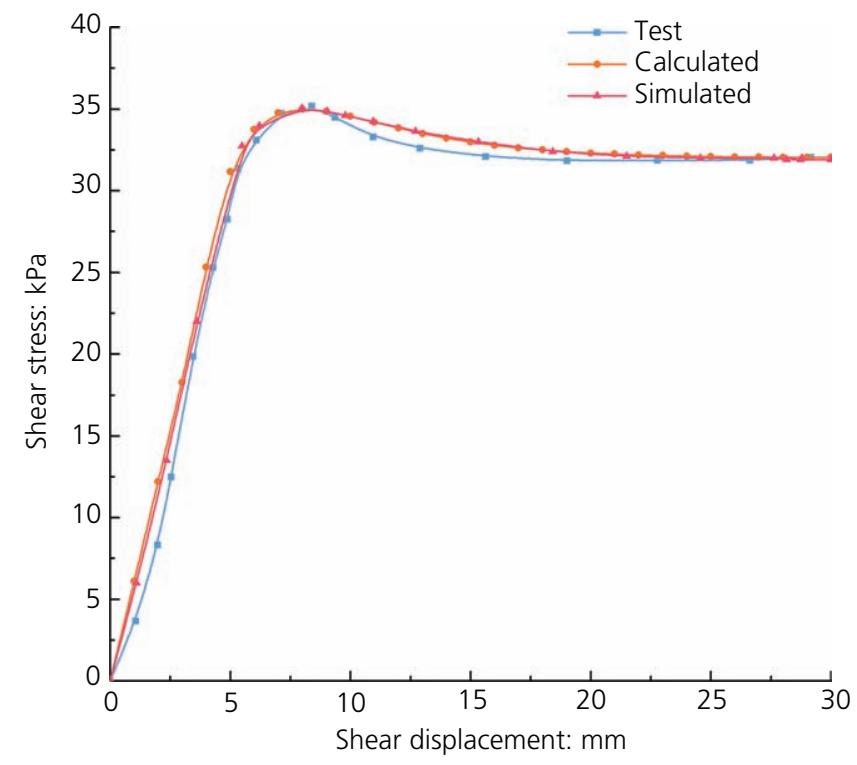

Figure 8. Comparison of the numerical results with experimental and theoretical calculation results

of the interface can be simulated, which proves the validity of the established FRIC subroutine.

\section{Normal displacement-shear displacement curve}

To analyse the variation of normal displacement of the upper sand body during the shear process, the normal displacement-shear displacement curve on both sides of the sand body top is extracted, as shown in Figure 9. With the increase of shear displacement, the phenomenon of dilatancy occurs at the top of

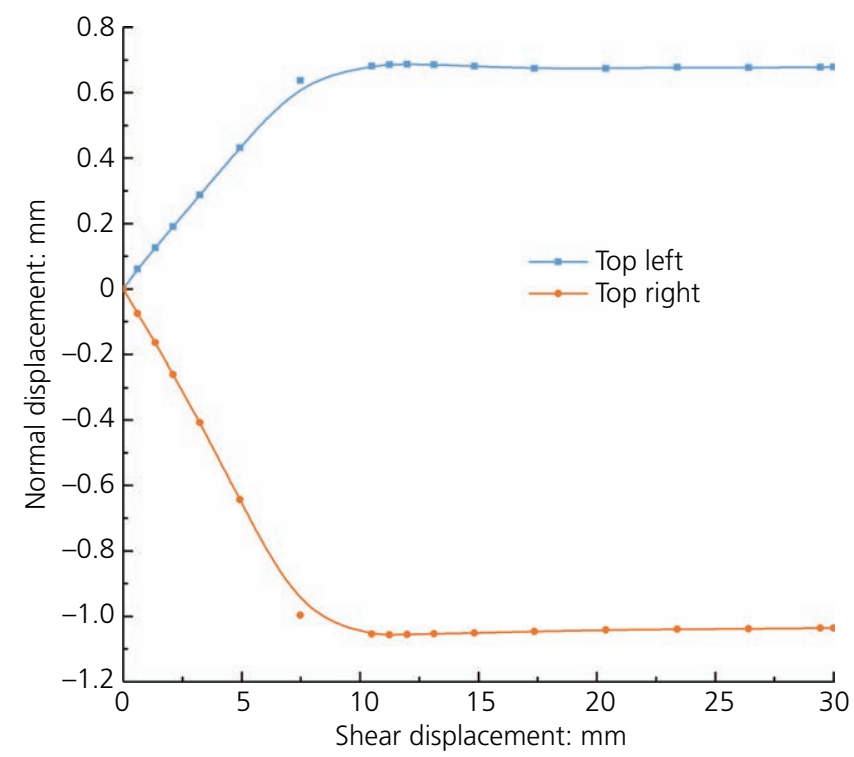

Figure 9. Relationship between normal displacement and shear displacement during shearing the left side of the sand, and the phenomenon of shear shrinkage occurs on the right side. The dilatancy on the left and the shrinkage on the right both increase rapidly in the early stage of shearing. As the shear displacement continues to increase, the amount of dilatancy and shrinkage tend to stabilise, and eventually no longer change. The normal displacements at different positions of the top of the sand after shearing are extracted, as shown in Figure 10. After the completion of the shear, the top of the sand from left to right appears as a phenomenon of shear expansion to shear shrinkage; the maximum shear expansion on the left side is $0.68 \mathrm{~mm}$ and the maximum shear shrinkage on the right side is $1.03 \mathrm{~mm}$. The main reason is that in the process of polymer moving from right to left, the particles on the left side of the sand are squeezed to form an extrusion area, and the particles on the right side become loose and form a loose zone. When the normal load is stable, shear expansion and shear expansion and right shear shrinkage will be left.

\section{Evolution law of interfacial shear stress}

During the shearing process, the interfacial shear stress continuously changes, and the evolution of the interfacial shear stress can be obtained by analysing the relationship of the shear stress during the shearing process. The results are shown in Figure 11.

When the shear displacement $s=0.6 \mathrm{~mm}$, the interface is in the elastic deformation stage, and the shear stress at both ends of the interface is greater than the shear stress in the middle part. The interface is divided into elastic zone and non-contact zone from left to right, as shown in Figure 11(a).

When the shear displacement $s=5 \mathrm{~mm}$, the interfacial shear stress continues to increase, and the increase range of shear stress on the

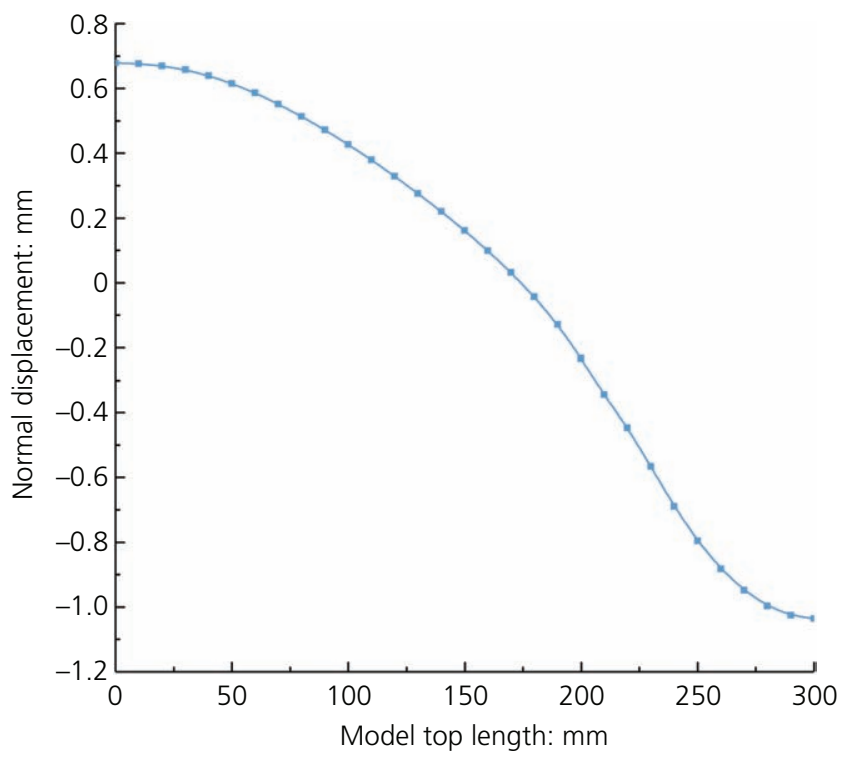

Figure 10. Normal displacement at different positions on top of the model 


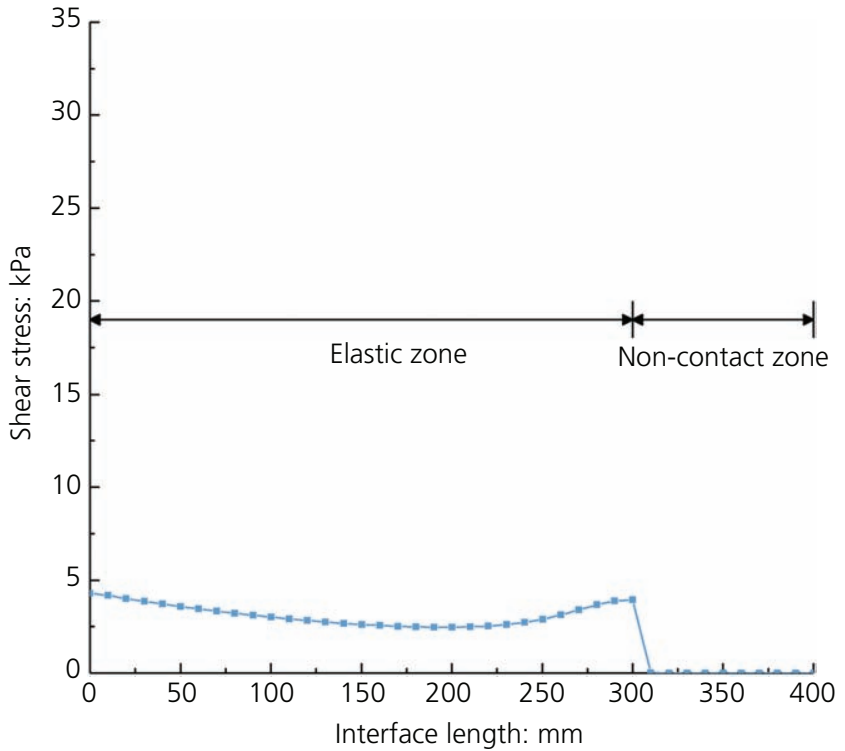

(a)

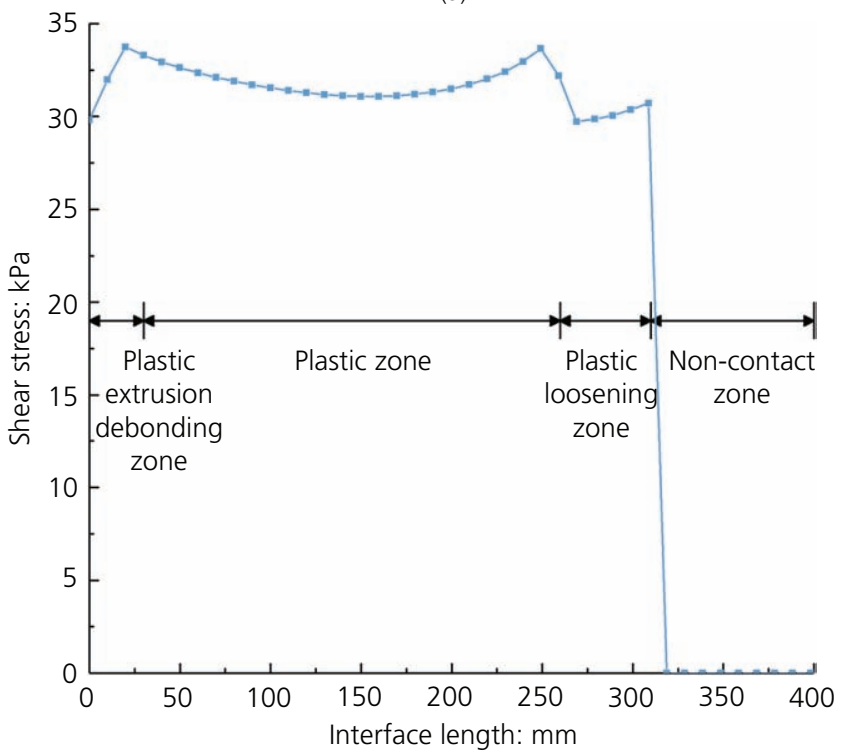

(c)

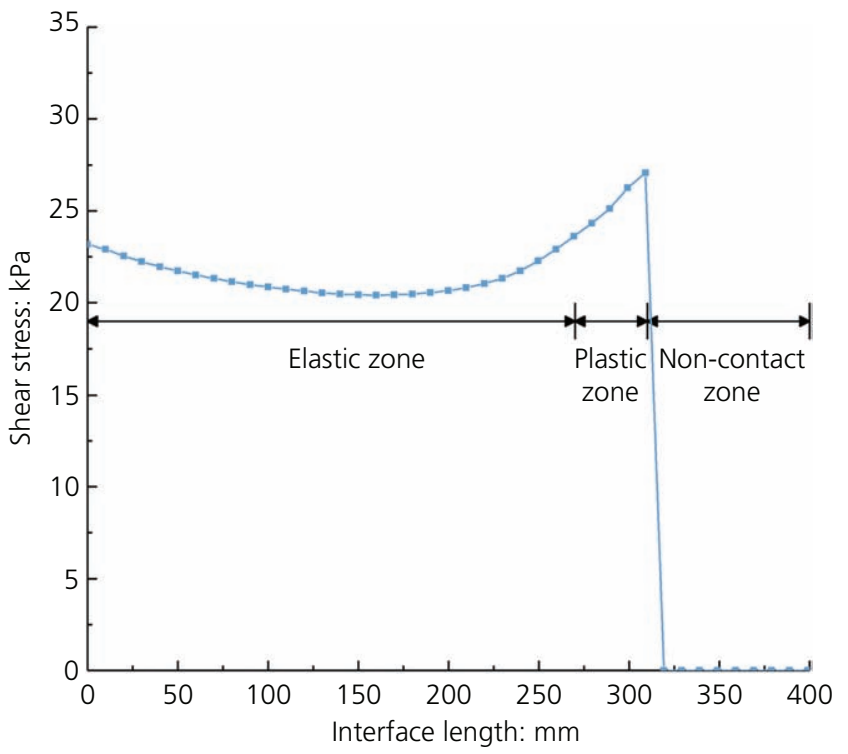

(b)

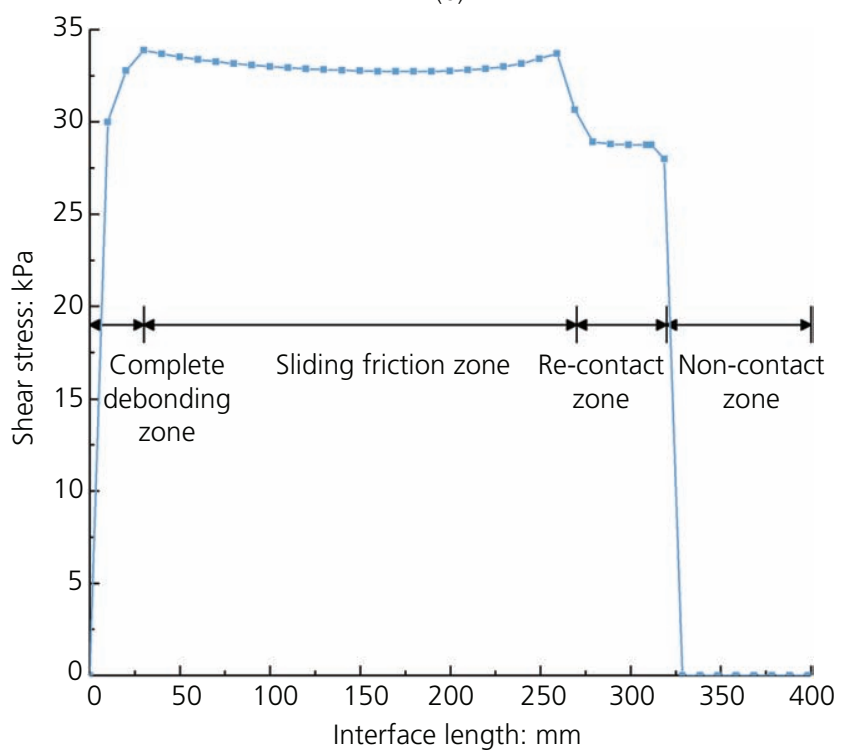

(d)

Figure 11. Evolution law of shear stress on interfaces: (a) $s=0.6 \mathrm{~mm}$; (b) $s=5 \mathrm{~mm}$; (c) $s=7.5 \mathrm{~mm}$; (d) $s=15 \mathrm{~mm}$

right side of the sand is greater than that on the left side. With the continuous increase of shear stress, plastic deformation occurs at the right end first. The interface is divided into elastic zone, plastic zone and non-contact zone from left to right, as shown in Figure 11(b).

When the shear displacement $s=7.5 \mathrm{~mm}$, with the continuous increase of shear stress, the plastic deformation of the interface further expands, and the bond strength on the left side of the interface is gradually destroyed, resulting in extrusion debonding. Due to the loosening of particles, the shear stress value in the right area becomes smaller. The interface is divided into plastic extrusion debonding zone, plastic zone, plastic loosening zone and non-contact zone from left to right, as shown in Figure 11(c).
When the shear displacement $s=15 \mathrm{~mm}$, the shear stress continues to increase and the interface bonding strength is completely destroyed. Then it enters the sliding friction stage, and the shear stress tends to be stable. As the shear displacement increases, complete debonding occurs on the left side of the interface, and the far-right side is in contact with the polymer again, but the shear stress value is less than the initial contact area, mainly because during the shearing process, the sand particles near the interface are adjusted to form a directional arrangement, and there is no bonding force in the re-contact area. The interface can be divided into complete debonding zone, sliding friction zone, re-contact zone and non-contact zone from left to right, as shown in Figure 11(d). 


\section{Conclusions}

Based on the analysis and summary of previous research results, this paper uses statistical damage and Weibull distribution theory to study the shear mechanical properties of strain-softening interfaces, derives the interface shear damage softening model and conducts detailed analysis on the parameter determination method. The numerical implementation method of the model is proposed, and the numerical verification is carried out according to the test data in the literature. The main conclusions are as follows.

- Based on statistical damage theory and Weibull distribution function, an interface shear damage softening model is established. The model contains five parameters, which can be obtained from the interface shear test, and the physical meaning of the parameters is clear. The determination method of parameters in the model is studied, and the relationship between parameters and normal stress is established, which lays the foundation for the universality of the model.

- Using the FRIC subroutine interface in Abaqus, the shear softening model of this paper is written into a subroutine, and numerical simulations are carried out based on the literature data. The results show that the model can reflect the interface shear stress-shear displacement relationship and shear softening characteristics.

- In this paper, the experimental data and numerical simulation results are used to verify the interface damage model, which proves the effectiveness of the model. The established numerical method provides convenience for engineering design and has practical value.

\section{Acknowledgements}

The authors are grateful for the financial support from the key research and development program of the Tibet Autonomous region (XZ201801-GB-07), the research and development program of China State Railway Group Co., Ltd. (K2019G032), and the Guangdong Program for Innovative Research Teams Introduction (2016ZT06N340).

\section{REFERENCES}

Cao W, Wang J and Zhai Y (2012) Study of simulation method for the shear deformation of rock structural planes and interfaces with consideration of residual strength. Chinese Journal of Theoretical and Applied Mechanics 45(4): 127-133 (in Chinese).

Chen J, Zhang J and Li J (2016) Influence of interface roughness on mechanical properties of red clay-concrete interface. Journal of Central South University (Science and Technology) 47(5): 1682-1688 (in Chinese).

Chen C, Leng W, Yang Q et al. (2018) Experimental study of mechanical properties of concrete pile-slurry-sand interface. Rock and Soil Mechanics 39(7): 2461-2472 (in Chinese).

Cheng H, Cheng X, Zhang J, Li J and Zhang B (2017) Experimental research on residual shear strength of red clay-concrete structure interface. Journal of Central South University (Science and Technology) 48(9): 2458-2464 (in Chinese).

Cheng H, Wang X, Zhang J and Song L (2018a) Shear behavior of geogrid-soil interface and its statistical damage softening model. Journal of Railway Science and Engineering 15(11): 2780-2787 (in Chinese).
Cheng H, Wang X, Zhang J and Wu Z (2018b) Effects of particle size on shear behavior of interface between coarse-grained soil and concrete. Journal of Basic Science and Engineering 26(1): 145-153 (in Chinese).

Clough G W and Duncan J M (1971) Finite element analyses of retaining wall behavior. ASCE Journal of the Soil Mechanics and Foundations Division 12(97): 1657-1673.

Desai CS and Ma YZ (1992) Modeling of joints and interfaces using the disturbed-state concept. International Journal for Numerical and Analytical Methods in Geomechanics 16(9): 623-653.

Hu Q, Jiang J, Xu Y, Shi R and Zhang Y (2017) Interface constitutive model test and numerical simulation of red mudstone pile-rock. Journal of Civil and Environmental Engineering 39(3): 122-128 (in Chinese).

Hu H, Chen X, Tang M, Liao X and Xiao Y (2018a) Investigation on shearing failure mechanism for DPC pile-soil interface in large-scale direct shear tests. Rock and Soil Mechanics 39(12): 4325-4334 (in Chinese).

Hu S, Li W, Wu J, Zhu F and Xu C (2018b) Research on mechanical properties of sand and steel interface by direct shear test. Industrial Construction 48(8): 118-121 (in Chinese).

Kong L, Xiong C, Guo A and Yang A (2017) Effects of shear rate on strength properties and pile-soil interface of marine soft clay. Chinese Journal of Geotechnical Engineering 39(S2): 13-16 (in Chinese).

Lemaitre J (1984) How to use damage mechanics. Nuclear Engineering and Design 80(2): 233-245.

Li Y, Wang W, Huang M and Guo Y (2015) Experimental research on pile-soil interface shear behaviors of super-long bored pile. Rock and Soil Mechanics 36(7): 1981-1988 (in Chinese).

Long Y, Chen J and Zhang J (2017) Introduction and analysis of a strainsoftening damage model for soil-structure interfaces considering shear thickness. KSCE Journal of Civil Engineering 21(7): 2634-2640.

Potyondy J G and Eng M (1961) Skin friction between various soils and construction materials. Geotechnique 4(11): 339-353.

Uesugi M and Kishida H (1986) Frictional resistance at yield between dry sand and mild steel. Soils and Foundations 4(26): 139-149.

Wang Y, Rao X, Pan J, Zuo Y and Gao P (2019a) Mechanical behaviors of interface between sand-gravel cushion material and concrete face slab by large-scale simple shear tests. Chinese Journal of Geotechnical Engineering 41(8): 1538-1544 (in Chinese).

Wang Y, Zhang M, Liu J and Bai X (2019b) Experimental research on shear characteristics of pile-soil interface in unsaturated clayey soil. Chinese Journal of Underground Space and Engineering 15(5): 1468-1474 (in Chinese).

Wang Y, Wan Y, Liu Q, Guo C and Shi M (2021) The study on interfacial shear properties of non-water reacted polymer and geomaterials. Journal of Building Materials 24(1): 115-120 (in Chinese).

Xiong B, Huang G and Xu W (2017) Experiment study on the effect of concrete roughness on shear behavior of pile-soil interface. Building Science 33(3): 39-45 (in Chinese).

Xiong B, Huang G, Xu W and Zhang Z (2018) Large-scale shear test on mechanical characteristics of interface between pile and soil. Journal of Nanjing Tech University (Natural Science Edition) 40(3): 67-72 (in Chinese).

Yin Z, Zhu H and Xu G (1994) Numerical simulation of the deformation in the interface between soil and structural material. Chinese Journal of Geotechnical Engineering 16(3): 14-22 (in Chinese).

\section{How can you contribute?}

To discuss this paper, please submit up to 500 words to the editor at journals@ice.org.uk. Your contribution will be forwarded to the author(s) for a reply and, if considered appropriate by the editorial board, it will be published as a discussion in a future issue of the journal. 